\title{
A STOCHASTIC DEVELOPMENT OF CLOUD COMPUTING BASED TASK SCHEDULING ALGORITHM
}

\author{
V. Karunakaran, \\ Department of Computer Sciences Technology, \\ Karunya Institute of Technology and Sciences, Coimbatore, India. \\ karunakaran@karunya.edu
}

\begin{abstract}
Due to diversity of services with respect to technology and resources, it is challenging to choose virtual machines (VM) from various data centres with varied features like cost minimization, reduced energy consumption, optimal response time and so on in cloud Infrastructure as a Service (IaaS) environment. The solutions available in the market are exhaustive computationally and aggregates multiple objectives to procure single trade-off that affects the solution quality inversely. This paper describes a hybrid algorithm that facilitates VM selection for scheduling applications based on Gravitational Search and Non-dominated Sorting Genetic Algorithm (GSA and NSGA). The efficiency of the proposed algorithm is verified by the simulation results.
\end{abstract}

Keywords: NSGA, Cloud Computing, GSA, Task Scheduling, Virtual Machine

\section{INTRODUCTION}

Cloud computing is a novel technology used for computing and enrich the dynamic user experience in accessing virtual resources and offer trustworthy and consistent services. Virtual resources that are scalable and shared such as services, storage and servers are provided by cloud computing. It is necessary to establish a proficient load balancing algorithm for effective and efficient utilization of cloud computing. This is the major objective of service providers. In order to meet the growing demand and to satisfy the principles of virtualization, it is essential to develop an improved task scheduling algorithm.

Task scheduling algorithm helps in meeting job requirements while reducing the completion period with the available resources, improving the load balance and achieving high system throughput. In normal task scheduling, the virtual machines are scheduled with a set of predefined number of tasks, whereas in cloud computing resources, since the user pays for the resources on time basis, task scheduling is of prime importance. The primary goals of cloud based scheduling schemes are minimizing task execution time, minimizing task transferring time, decreasing task execution cost, reducing makespan, ensuring user-level QoS in task execution by meeting the 
SLA, increasing elasticity, increasing resource availability, balancing of load and reduces energy consumption $[2]$.

This paper recommends a task scheduling algorithm which is energy efficient, can calculate the total utilization as well as completion time of tasks and make scheduling decisions by normalizing them. In comparison with the existing algorithms such as Dynamic Cloud List Scheduling (DCLS), Round Robin (RR), Energy-Aware Task Consolidation (ETC), MaxUtil and Energy-Conscious Task Consolidation (ECTC), the proposed algorithm offers better performance and efficiency.

It provides a combination of Green Supply Chain Management with a combination of Fuzzy Set Theory and Grey Theory, Green Product Decision and Deletion with Neighbourhood Rough Set Theory (NRSI), Fuzzy Cluster Means (FCM) and Cumulative Prospect Theory (CPT) and Green cradle to cradle performance evaluation system. We use synthetic datasets and modified benchmarks to perform an extensive simulation of the algorithm. Implementation and assessment of simulation results with suggested algorithm and evaluation of energy consumption and makespan of the algorithm.

\section{EXISTING LITERATURE}

Ketaki Naik et al [2] performed a complete analysis of the cloud computing scheduling schemes that are based on Particle Swarm Optimization (PSO). These schemes are classified based on the algorithm type and further analysis is done elaborating the improvement of PSO algorithm in each scheme and its integration to provide solutions to scheduling issues. Execution time, cost, load balancing, makespan and other factors are also compared for these schemes.

In cloud Software as a Service (SaaS) environment, Atul Vikas et al [3] proposed a task scheduling algorithm that is multi-objective and satisfies the SLA (Service Level Agreement) while reducing the cost and improving the data centre throughput when mapping tasks to VMs. When traditional algorithms perform task scheduling considering a single criteria, the proposed algorithm in this paper provides optimal scheduling and considers multiple criteria such as cost, user bandwidth, and execution time and so on.

Syed Hamid et al [4] recommended adoption of heuristic algorithm in IaaS environment as a standard for comparison for an algorithm that can resolve and enhance task scheduling and other related issues. Heuristic algorithms are simple and easy to implement and thus provides optimal and rapid outcome. Further optimization can be achieved in IaaS environment by combining meta-heuristic algorithm along with heuristic algorithm as they complement each other overcoming mutual drawbacks. 
Nima Jafari et al [5] presented the Cuckoo Search Algorithm (CSA) for task scheduling in cloud computing environment. This technology combines the flight behaviour of fruit flies and some birds along with the obligate brood parasitic behaviour found in certain cuckoo species. When the probability of the ' $n$ ' nest 'pa' value is low, the algorithm offers high coverage and speed. Jocksam et al [6] proposed a solution to reduce processing time in task scheduling and resolve partitioning problem by using a combination of metaheuristic genetic algorithm (GA) and static algorithm.

Arunarani et al [8] performed a survey of over 60 available task scheduling techniques highlighting their time complexity, limitations, reliability and availability. Every method has its own pros and cons and works on diverse applications like load balancing, resource allocation and task scheduling. Every algorithm presented has its own efficiency and provides scope for enhancement. Certain notable limitations in these algorithm includes delay, computational complexity, overloading and maximum scheduling time. The major techniques used in these algorithm includes QoS, Ant, PSO, GA, ACO, Fuzzy, clustering and so on.

\section{PROPOSED WORK}

Task scheduling and selection of virtual machines are the major functions in optimization when dealing with multi-objective processes. The processing speed of the task is represented by $\mathrm{p}$, bandwidth by s, storage space by $\mathrm{r}$ and memory size by q. Figure 1 represents the chromosome encoding. For optimization of the genetic operators, we consider mutation, crossover and selection.

\begin{tabular}{|l|l|l|l|l|l|l|l|}
\hline T1 & T2 & T3 & T4 & T5 & T6 & T7 & T8 \\
\hline 1 & 4 & 2 & 3 & 3 & 1 & 1 & 4 \\
\hline
\end{tabular}

Figure 1: Chromosome encoding

Table 1: Computation of Energy Consumption using GSA and NGSA algorithm:

\begin{tabular}{|l|l|l|}
\hline & \multicolumn{1}{|c|}{ GSA Algorithm } & \multicolumn{1}{c|}{ NSGA Algorithm } \\
\hline Step 1 & $\begin{array}{l}\text { Produce N virtual machines based Initial } \\
\text { Population }\end{array}$ & $\begin{array}{l}\text { Produce Preliminary population arbitrarily and by } \\
\text { GSA }\end{array}$ \\
\hline Step 2 & $\begin{array}{l}\text { Compute fitness of every individual VM } \\
\text { using } \\
E C=\sum_{j=1}^{m} \sum_{i=1}^{n} e_{i j} \times x_{i j} \\
\text { Where } \mathrm{e}_{\mathrm{ij}} \text { denotes energy consumption while } \\
\text { execution of task i on } \mathrm{VM} \mathrm{j} .\end{array}$ & Compute objective fitness of every individual \\
\hline
\end{tabular}




\begin{tabular}{|c|c|c|}
\hline Step 3 & $\begin{array}{l}\text { Compute Best and Worst fitness value using } \\
\qquad \operatorname{worst}(t)=\max _{b=1}^{N}\left\{f_{\mathrm{b}}(t)\right\} \\
\qquad \operatorname{best}(t)=\min _{b=1}^{N}\left\{f i t_{b}(t)\right\} \\
\text { where } \mathrm{t} \text { is time (iteration), fit }{ }_{\mathrm{b}} \text { denotes the } \\
\text { fitness value of the bth particle, } \mathrm{M}_{\mathrm{b}} \text { is } \\
\text { the normalized mass and } \mathrm{N} \text { is the number of } \\
\text { particles }\end{array}$ & $\begin{array}{l}\text { Crossover is applied on parent and generate a new } \\
\text { population of the similar size }\end{array}$ \\
\hline Step 4 & Apprise Gravitational Constant $G$ & $\begin{array}{l}\text { Apply mutation on the new population and revise } \\
\text { the new population. }\end{array}$ \\
\hline Step 5 & $\begin{array}{l}\text { Estimate mass of individual virtual machine } \\
\text { using } \\
m_{b}(t)=\frac{\operatorname{fit}_{b}(t)-\operatorname{worst}(t)}{\operatorname{best}(t)-\operatorname{worst}(t)} b=1,2 \ldots . N \\
\qquad M_{b}(t)=\frac{m_{b}(t)}{\sum_{c=1}^{N} m_{c}(t)} 0 \leq M_{b}(t)<1\end{array}$ & $\begin{array}{l}\text { Select the greater individuals from the parent and } \\
\text { the new population to form the offspring with the } \\
\text { individuals }\end{array}$ \\
\hline Step 6 & $\begin{array}{l}\text { Estimate acceleration matrix using } \\
a_{b}^{d}(t)=\frac{F_{b}^{d}(t)}{M_{b}(t)}\end{array}$ & Review the Pareto front solution \\
\hline Step 7 & $\begin{array}{l}\text { Estimate the velocity and positions of virtual } \\
\text { machines using } \\
\qquad \begin{array}{l}V_{b}^{d}(t+1)=r_{b} \times V_{b}^{d}(t)+a_{b}^{d}(t) \\
\qquad X_{b}^{d}(t+1)=x_{b}^{d}(t)+V_{b}^{d}(t+1)\end{array}\end{array}$ & Go to step 2 \\
\hline Step 8 & Repeat until stop condition met & - \\
\hline
\end{tabular}

The GSA search space contains particle sets termed as masses that offers solution to optimization issue and is located as a point in space. The NSGA can perform exploration by expanding the search space. The major characteristics of this algorithm are low computational complexity, parameter-less diversity preservation and elitism. The combination of these algorithms and the features allow achievement of cost reduction and minimization of response time with reduced energy consumption. CloudSim simulation toolkit is used for simulation purpose. It facilitates features like scheduling policies, virtual machine and data centre.

\section{RESULT}

We use a cloud simulator for the purpose of setting the parameters. Virtual Machines, Microprocessor without Interlocked Pipelined Stages (MIPS), Virtual RAM, bandwidth, processing elements required and so on are predefined and represented in the table 2. The analysis is done for both homogeneous as well as heterogeneous environment. Comparison of algorithms like Sufferage, Min-min, Max-min, Minimum Execution Time (MET), Minimum Completion Time (MCT) and First Come First Serve (FCFS) are done. The cost, degree of imbalance, 
Journal of Soft Computing Paradigm (JSCP) (2019)

Vol.01/ No. 01

Pages: $41-48$

https://irojournals.com/jscp/

DOI: https://doi.org/10.36548/jscp.2019.1.005

makespan and throughput factors are analysed in homogenous environment. The cost based on HPC2N and NASA datasets, left-skewed, right-skewed, normal and uniform distribution are analysed in heterogeneous environment.

Table 2 Cloud Simulator parameter initialization

\begin{tabular}{|l|l|l|}
\hline \multicolumn{1}{|c|}{ Type } & \multicolumn{1}{c|}{ Parameter } & \multicolumn{1}{c|}{ Value } \\
\hline Data Center & Host count & $8-10$ \\
\hline User & Count & 20 \\
\hline Virtual Machine & Virtual Machine Count & 80 \\
\cline { 2 - 3 } & Policy type & Time Share \\
\cline { 2 - 3 } & MIPS & $500-4000$ \\
\cline { 2 - 3 } & RAM & $1024-2048 \mathrm{MB}$ \\
\cline { 2 - 3 } & Bandwidth & $500-1000 \mathrm{~b}$ \\
\cline { 2 - 3 } & OS & Linux \\
\hline Tasks & Task count & $2500-4000$ \\
\cline { 2 - 3 } & Task length & $4-15$ \\
\cline { 2 - 3 } & PE count & MI \\
\hline
\end{tabular}

\section{Response Time vs. Cost}

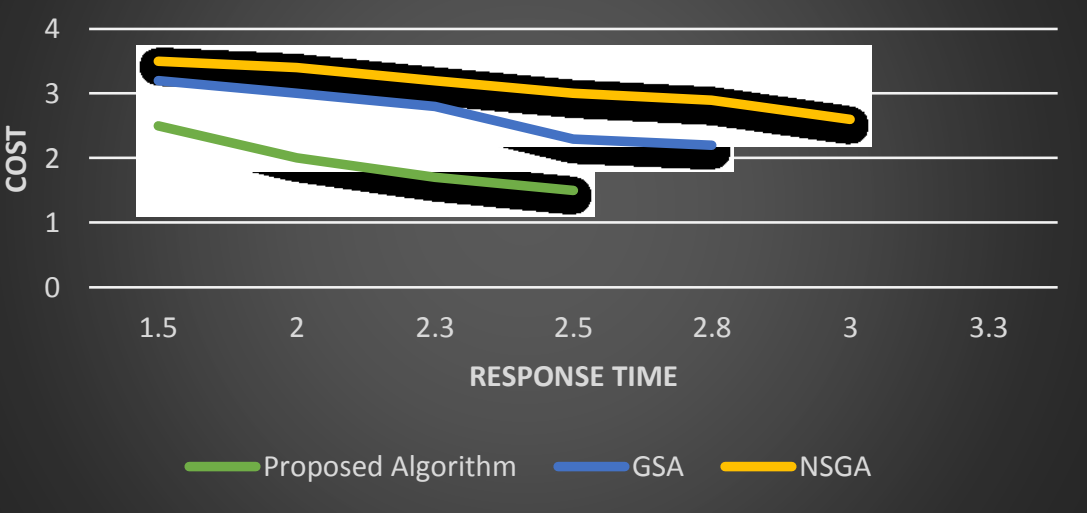




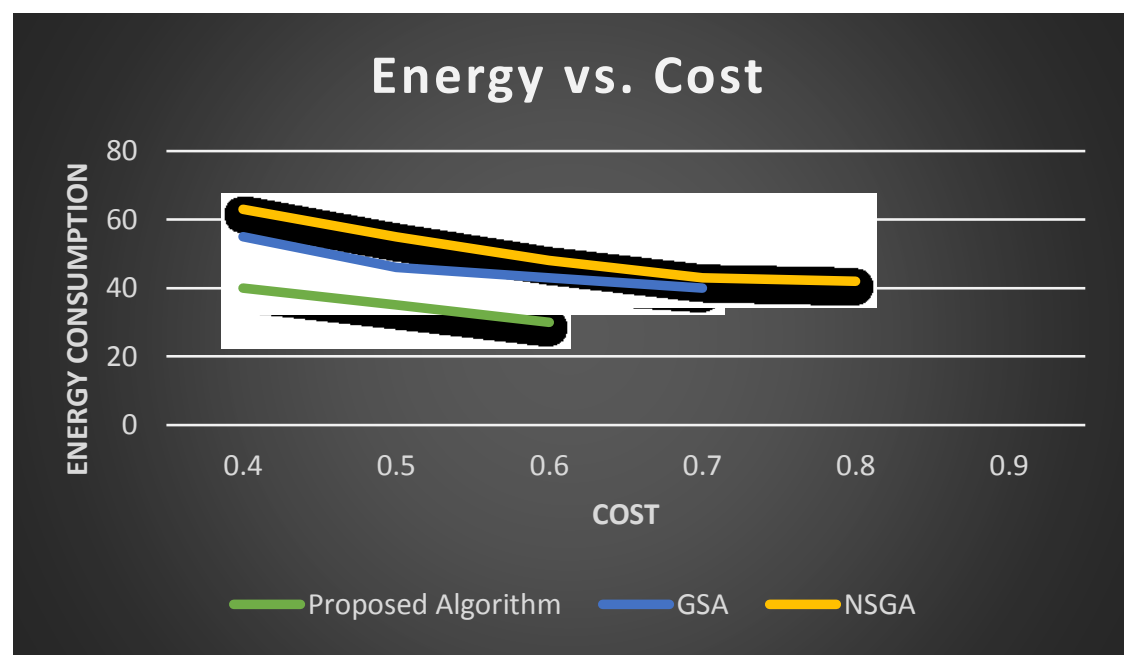

Figure 3: Energy consumption vs. Cost comparison

The major factors considered here are energy consumption, cost and response time. From the above graphs, it is shown that the proposed algorithm offers optimal solution in all the three areas efficiently. The utilization and completion time provides the minimum normalized value total. Mapping of every individual task is done with all the resources prior to assigning the task. Makespan and energy mitigation is done as the algorithm emphasizes utilization value as well as completion time.

\section{CONCLUSION AND FUTURE SCOPE}

In IaaS environment, the proposed method offers efficiency and improved performance in consideration of energy consumption, execution cost and response time. This system overcomes the drawbacks like computational exhaustiveness and multiple objective aggregation of the existing systems. The GSA algorithm performs shortlisting of virtual machines with low power consumption whereas the NSGA assists in selection of VM with low execution cost and response time. Simulation results prove the efficiency of the proposed algorithm.

Heuristic algorithm are easy to implement and are simple. This feature makes the algorithm faster and provide optimal outputs. It is essential to ensure minimal execution time for the overall algorithm. The algorithm offers strong global as well as local search capabilities and offers faster convergence of the outputs. Future work involves power consumption reduction in cloud data centres, implementation of green cloud computing and load balancing. 


\section{References}

[1] Al-Maamari, Ali, and Fatma A. Omara. "Task scheduling using PSO algorithm in cloud computing environments." International Journal of Grid and Distributed Computing 8, no. 5 (2015): 245-256.

[2] Masdari, Mohammad, Farbod Salehi, Marzie Jalali, and Moazam Bidaki. "A survey of PSO-based scheduling algorithms in cloud computing." Journal of Network and Systems Management 25, no. 1 (2017): 122-158.

[3] Lakra, Atul Vikas, and Dharmendra Kumar Yadav. "Multi-objective tasks scheduling algorithm for cloud computing throughput optimization." Procedia Computer Science 48 (2015): 107-113.

[4] Madni, Syed Hamid Hussain, Muhammad Shafie Abd Latiff, Mohammed Abdullahi, and Mohammed Joda Usman. "Performance comparison of heuristic algorithms for task scheduling in IaaS cloud computing environment." PloS one 12, no. 5 (2017): e0176321.

[5] Navimipour, Nima Jafari, and Farnaz Sharifi Milani. "Task scheduling in the cloud computing based on the cuckoo search algorithm." International Journal of Modeling and Optimization 5, no. 1 (2015): 44.

[6] Matos, Jocksam G. De, Carla K. De M. Marques, and Carlos HP Liberalino. "Genetic and static algorithm for task scheduling in cloud computing." International Journal of Cloud Computing 8, no. 1 (2019): 1-19.

[7] Panda, Sanjaya K., and Prasanta K. Jana. "An energy-efficient task scheduling algorithm for heterogeneous cloud computing systems." Cluster Computing 22, no. 2 (2019): 509-527.

[8] Arunarani, A. R., D. Manjula, and Vijayan Sugumaran. "Task scheduling techniques in cloud computing: A literature survey." Future Generation Computer Systems 91 (2019): 407-415.

[9] Gamal, Marwa, Rawya Rizk, Hani Mahdi, and Basem Elhady. "Bio-inspired Based Task Scheduling in Cloud Computing." In Machine Learning Paradigms: Theory and Application, pp. 289-308. Springer, Cham, 2019.

[10] Yiqiu, Fang, Xiao Xia, and Ge Junwei. "Cloud Computing Task Scheduling Algorithm Based On Improved Genetic Algorithm." In 2019 IEEE 3rd Information Technology, Networking, Electronic and Automation Control Conference (ITNEC), pp. 852-856. IEEE, 2019.

[11] Kashikolaei, Seyedeh Monireh Ggasemnezhad, Ali Asghar Rahmani Hosseinabadi, Behzad Saemi, Morteza Babazadeh Shareh, Arun Kumar Sangaiah, and Gui-Bin Bian. "An enhancement of task scheduling in cloud computing based on imperialist competitive algorithm and firefly algorithm." The Journal of Supercomputing (2019): 1-28.

[12] Wang, Qing, Xue-Liang Fu, Gai-Fang Dong, and Tao Li. "Research on cloud computing task scheduling algorithm based on particle swarm optimization." Journal of Computational Methods in Sciences and Engineering Preprint (2019): 1-9.

[13] Maguluri, Siva Theja, Rayadurgam Srikant, and Lei Ying. "Stochastic models of load balancing and scheduling in cloud computing clusters." In 2012 Proceedings IEEE Infocom, pp. 702-710. IEEE, 2012.

[14] Guo, Lizheng, Shuguang Zhao, Shigen Shen, and Changyuan Jiang. "Task scheduling optimization in cloud computing based on heuristic algorithm." Journal of networks 7, no. 3 (2012): 547. 
[15] Zhang, Luna Mingyi, Keqin Li, and Yan-Qing Zhang. "Green task scheduling algorithms with speeds optimization on heterogeneous cloud servers." In Proceedings of the 2010 IEEE/ACM Int'l Conference on Green Computing and Communications \& Int'l Conference on Cyber, Physical and Social Computing, pp. 76-80. IEEE Computer Society, 2010.

[16] Zhou, Zhou, Fangmin Li, Huaxi Zhu, Houliang Xie, Jemal H. Abawajy, and Morshed U. Chowdhury. "An improved genetic algorithm using greedy strategy toward task scheduling optimization in cloud environments." Neural Computing and Applications (2019): 1-11.

[17] Jana, Bappaditya, Moumita Chakraborty, and Tamoghna Mandal. "A task scheduling technique based on particle swarm optimization algorithm in cloud environment." In Soft Computing: Theories and Applications, pp. 525-536. Springer, Singapore, 2019.

[18] Abazari, Farzaneh, Morteza Analoui, Hassan Takabi, and Song Fu. "MOWS: multi-objective workflow scheduling in cloud computing based on heuristic algorithm." Simulation Modelling Practice and Theory 93 (2019): 119-132.

[19] Abdullahi, Mohammed, Md Asri Ngadi, Salihu Idi Dishing, and Barroon Isma'eel Ahmad. "An efficient symbiotic organisms search algorithm with chaotic optimization strategy for multi-objective task scheduling problems in cloud computing environment." Journal of Network and Computer Applications 133 (2019): 60-74.

[20] Geng, Xiaozhong, Lan Yu, Jie Bao, and Geji Fu. "A task scheduling algorithm based on priority list and task duplication in cloud computing environment." In Web Intelligence, vol. 17, no. 2, pp. 121-129. IOS Press, 2019.

[21] Panda, Sanjaya K., Indrajeet Gupta, and Prasanta K. Jana. "Task scheduling algorithms for multi-cloud systems: allocation-aware approach." Information Systems Frontiers 21, no. 2 (2019): 241-259.

[22] Li, Jian-Feng, and Jian Peng. "Task scheduling algorithm based on improved genetic algorithm in cloud computing environment." Jisuanji Yingyong/ Journal of Computer Applications 31, no. 1 (2011): 184-186.

[23] Salot, Pinal. "A survey of various scheduling algorithm in cloud computing environment." International Journal of Research in Engineering and Technology 2, no. 2 (2013): 131-135. 